\title{
Magnetic tuning of ultracold barrierless chemical reactions
}

\author{
Timur V. Tscherbul ${ }^{1}$ and Jacek Kłos ${ }^{2}$ \\ ${ }^{1}$ Department of Physics, University of Nevada, Reno, Nevada 89557, USA \\ ${ }^{2}$ Department of Chemistry and Biochemistry, University of Maryland, College Park, Maryland 20742, USA
}

(Received 27 April 2019; revised manuscript received 21 November 2019; accepted 18 December 2019; published 3 February 2020)

\begin{abstract}
While attaining external field control of bimolecular chemical reactions has long been a coveted goal of physics and chemistry, the role of hyperfine interactions and dc magnetic fields in achieving such control has remained elusive. We develop an extended coupled-channel statistical theory of barrierless atom-diatom chemical reactions, and apply it to elucidate the effects of magnetic fields and hyperfine interactions on the ultracold chemical reaction $\operatorname{Li}\left({ }^{2} S_{1 / 2}\right)+\mathrm{CaH}\left({ }^{2} \Sigma^{+}\right) \rightarrow \mathrm{LiH}\left({ }^{1} \Sigma^{+}\right)+\mathrm{Ca}\left({ }^{1} S_{0}\right)$ on a newly developed set of $a b$ initio potential energy surfaces. We observe large field effects on the reaction cross sections, opening up the possibility of controlling ultracold barrierless chemical reactions by tuning selected hyperfine states of the reactants with an external magnetic field.
\end{abstract}

DOI: 10.1103/PhysRevResearch.2.013117

\section{INTRODUCTION}

Using external electromagnetic fields to control chemical reactivity is a central goal of chemical physics [1,2], which stimulated the development of new research avenues ranging from mode-selective chemistry [1] and coherent control [2] to the study of stereodynamics and vector correlations in molecular collisions [3-5] and ultracold controlled chemistry [6,7]. Molecular chemical reactions are most readily controlled at ultralow temperatures, where the reactants can be prepared in single internal and motional quantum states [8], which maximizes the effects of external electromagnetic fields [9] and allows for the manifestation of quantum phenomena, which would otherwise be obscured by thermal averaging, such as threshold and resonance scattering $[7,8,10]$, tunneling $[7,11]$, and interference $[12,13]$. Recent examples include the observation of resonance scattering in low-temperature $\mathrm{He}^{*}+$ $\mathrm{H}_{2}$ [10], $\mathrm{He}+\mathrm{NO}$ [14], and $\mathrm{NO}+\mathrm{H}_{2}$ [15] collisions, stereodynamical control of low-temperature $\mathrm{H}_{2}+\mathrm{HD}$ collisions in merged molecular beams [4,5], and chemical reactions in trapped ensembles of alkali-metal dimers [16,17] and atomdimer mixtures [18]. The vast majority of the previous control studies have focused on the rovibrational and nuclear spin degrees of freedom of the reactants. In particular, the chemical reaction $\mathrm{KRb}+\mathrm{KRb} \rightarrow \mathrm{K}_{2}+\mathrm{Rb}_{2}$ can be efficiently suppressed by preparing the reactants in the same rotational and nuclear spin states [19] and stimulated by applying an external electric field, which modifies the $p$-wave centrifugal barrier preventing the reaction of two identical fermionic molecules $[16,20]$. Several groups used external magnetic

Published by the American Physical Society under the terms of the Creative Commons Attribution 4.0 International license. Further distribution of this work must maintain attribution to the author(s) and the published article's title, journal citation, and DOI. fields to control chemical reactivity by tuning the orientation and fine-structure populations of the reactants [21-23].

Recent experimental advances in laser cooling and trapping $[24,25]$ have led to the production of dense, trapped ensembles of molecular radicals (i.e., molecules with nonzero electron spins) such as $\left.\mathrm{CaF}^{2}{ }^{2} \Sigma^{+}\right)$[26-29], $\operatorname{SrF}\left({ }^{2} \Sigma^{+}\right)$[30], $\mathrm{YbF}\left({ }^{2} \Sigma^{+}\right)$ [31], and $\operatorname{SrOH}\left({ }^{2} \Sigma^{+}\right)$[32]. Cotrapping of these molecules with ultracold alkali-metal atoms [30,31] would open up the fascinating prospect of studying spin-selective ultracold controlled chemistry $[6,33,34]$. Specifically, the electron spins of the reactants can be polarized in an external magnetic field to form a nearly spin-pure state in the entrance reaction channel corresponding to, e.g., the maximum possible total spin $S$ of the reaction complex $[6,33]$. Because such high- $S$ states are typically nonreactive, the chemical reaction of spin-aligned reactants $[A(\uparrow)+B(\uparrow)]$ will be suppressed compared to that of spin-antialigned reactants $[A(\downarrow)+B(\uparrow)]$.

However, theoretical studies of the effects of spin polarization, hyperfine interactions, and external magnetic fields on atom-molecule chemical reactions have been limited to reactions of weakly bound Feshbach molecules [35,36], save for a recent model study of ultracold NH-NH reactive scattering in a magnetic field [37], which did not include the hyperfine structure of $\mathrm{NH}$ and focused on collisions of fully spin-polarized molecules. As a result, the effects of hyperfine interactions and magnetic fields on ultracold reaction dynamics remain unexplored, limiting our ability to use the fields as a tool to control chemical reactivity at ultralow temperatures.

Here, we develop a theoretical approach to ultracold reaction dynamics in a magnetic field based on a rigorous coupledchannel statistical (CCS) model [38-41]. The CCS model postulates the existence of a long-lived reaction complex formed temporarily when the reactants get trapped in a long-lived resonance state [38-40,42,43], a powerful idea that forms the basis for quantum threshold models $[44,45]$ and quantum defect theories [46-49]. The CCS approach rigorously accounts for the multichannel nature of the molecular wave function in the entrance and exit reaction channels [38-40] and it has been 
successfully applied to calculate low-temperature inelastic [50] and reactive [51-53] collision rates. Unlike the universal model of chemical reactions [44-49], the CCS model can generate fully state-to-state reaction probabilities [38-40], albeit at a higher computational cost [51,52]. Building on the previous work, we extend the CCS approach to explicitly include the effects of hyperfine interactions and external magnetic fields in the entrance reaction channel, which allows us to explore the magnetic field dependence of the reaction cross sections. We exemplify the extended CCS approach by applying it to the chemical reaction $\mathrm{Li}+\mathrm{CaH} \rightarrow \mathrm{LiH}+\mathrm{Ca}$ on a newly developed set of ab initio potential energy surfaces (PESs). Our field-free results are in good agreement with experiment at $T=1 \mathrm{~K}$ [54]. We find that the reaction can be efficiently suppressed by tuning the hyperfine states of the reactants with an external magnetic field, opening up the possibility for controlling ultracold spin-dependent chemical reactions. Minimizing atom-molecule reaction rates is essential for efficient sympathetic cooling, in which molecules are immersed in a gas of ultracold atoms and refrigerated by elastic collisions [55-58]. Our results thus show that sympathetic cooling of chemically reactive ${ }^{2} \Sigma$ radicals could be facilitated by applying external magnetic fields.

\section{THEORY}

The original CCS theory $[38,39]$ relates the state-to-state reaction probability $P_{\gamma_{A} \gamma_{B} \rightarrow \gamma_{A}^{\prime} \gamma_{B}^{\prime}}$ to the capture probabilities in the entrance and exit reaction channels $p_{\gamma_{A} \gamma_{B}}$ and $p_{\gamma_{A}^{\prime} \gamma_{B}^{\prime}}$ as $P_{\gamma_{A} \gamma_{B} \rightarrow \gamma_{A}^{\prime} \gamma_{B}^{\prime}}=p_{\gamma_{A} \gamma_{B}} p_{\gamma_{A}^{\prime} \gamma_{B}^{\prime}} / \mathcal{N}$, where $\gamma_{A}$ and $\gamma_{B}$ refer to the incident rovibrational and hyperfine states of the reactants (molecule $A$ and atom $B$ ), and $\mathcal{N}=\sum_{\gamma_{A}^{\prime} \gamma_{B}^{\prime}} p_{\gamma_{A}^{\prime} \gamma_{B}^{\prime}}$ is a normalization factor. To obtain the capture probabilities $p_{\gamma_{A} \gamma_{B}}$, we solve the Schrödinger equation for the atom-molecule reaction complex described by the Hamiltonian (in atomic units, where $\hbar=1)[38-40,59]$

$$
\hat{H}=-\frac{1}{2 \mu R} \frac{\partial^{2}}{\partial R^{2}} R+\frac{\hat{L}^{2}}{2 \mu R^{2}}+\hat{V}(\boldsymbol{R}, \boldsymbol{r})+\hat{H}_{A}+\hat{H}_{B}
$$

subject to capture boundary conditions [38-40] as described in Appendix A. Here, $\boldsymbol{R}$ is the atom-molecule separation vector, $\boldsymbol{r}$ joins the nuclei in the diatomic molecule, and $\mu$ and $\hat{L}$ are the reduced mass and orbital angular momentum of the collision complex, respectively. The asymptotic Hamiltonians $\hat{H}_{A}$ and $\hat{H}_{B}$ account for the rotational, fine, and hyperfine structure of the reactants in the presence of an external magnetic field (see Appendix A), which is crucial for controlling ultracold reaction dynamics, as shown below.

The atom-molecule interaction operator in Eq. (1) is given by $\hat{V}(\boldsymbol{R}, \boldsymbol{r})=\sum_{S, M_{S}} V_{S}(R, r, \theta)\left|S M_{S}\right\rangle\left\langle S M_{S}\right|$, where $V_{S}(R, r, \theta)$ are the adiabatic atom-molecule PESs in the entrance reaction channel calculated $a b$ initio as described in Appendix B, $S$ is the total spin of the reaction complex, and $M_{S}$ is the projection of $S$ on the magnetic field axis. Figure 1(a) shows that both the singlet and triplet PESs are strongly anisotropic. The global minimum of the singlet PES is about twice as deep as that of the triplet PES. The approach of $\mathrm{Li}$ from the $\mathrm{Ca}$ side of $\mathrm{CaH}\left(\theta=180^{\circ}\right)$ is much more energetically favorable on the singlet PES, which has a deep local minimum in the linear configuration.

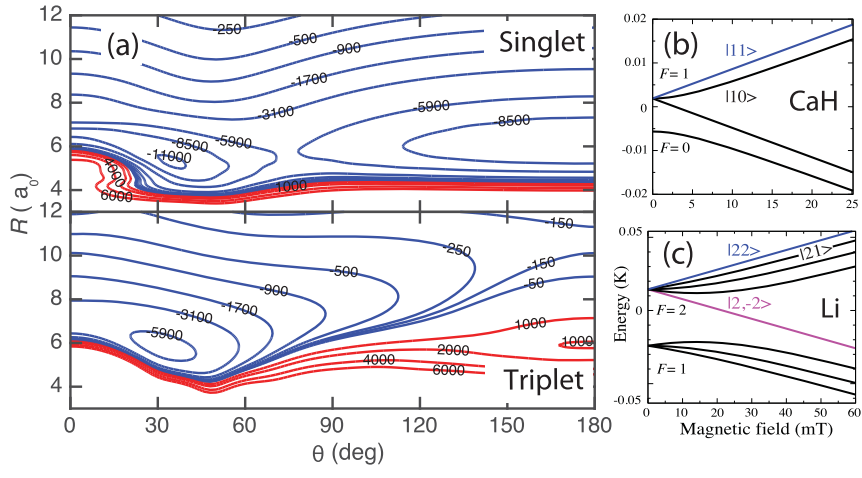

FIG. 1. (a) Contour plots of the ab initio Li-CaH PES of singlet ( $S=0$, top) and triplet ( $S=1$, bottom) symmetries. (b), (c) Zeeman energy levels of $\mathrm{CaH}$ and $\mathrm{Li}$. The initial hyperfine states used in our CCS calculations are labeled as $\left|F_{i} m_{F_{i}}\right\rangle(i=A, B)\left(F_{i}\right.$ is an approximate quantum number at $B>0$ ).

To solve the quantum reactive scattering problem in the presence of an external magnetic field, we expand the eigenfunctions of the Hamiltonian (1) in eigenstates of the total angular momentum of the reaction complex $|J M \Omega\rangle$ multiplied by the eigenstates of the fragment Hamiltonians $\hat{H}_{A}$ and $\hat{H}_{B}$. The resulting coupled-channel (CC) equations are solved numerically as described in Appendix A by initializing the complex multichannel log-derivative matrix $\mathbf{Y}$ [40] at the capture radius $R_{c}$ corresponding to the formation of the reaction complex, $\mathbf{Y}\left(R_{c}\right)=\mathbf{C}\left(R_{c}\right) \mathbf{Y}_{d}\left(R_{c}\right) \mathbf{C}^{T}\left(R_{c}\right)$. Here, $\mathbf{C}\left(R_{c}\right)$ are the eigenvectors of the potential coupling matrix, and $\mathbf{Y}_{d}\left(R_{c}\right)$ is the diagonal eigenvalue matrix initialized using the Airy boundary conditions. Having specified the initial value of $\mathbf{Y}$, we propagate the log-derivative matrix out to a large value of $R$ in the asymptotic region. The matrix elements of the Hamiltonian (1) are evaluated as described in our previous work [59] with the following essential modifications: (1) both the singlet and triplet PES of $\mathrm{Li}-\mathrm{CaH}$ are included in CCS calculations (see Appendix B); (2) the singlet PES is modified at $R=R_{m}$ to account for its reactive nature (the results of the calculations are largely insensitive to $R_{m}$ as shown in Appendix B; (3) the hyperfine degrees of freedom of the reactants are explicitly included, as are their interactions with an external magnetic field (see Appendix A). The final outcome of the calculations is the scattering $S$ matrix, which defines the reaction and capture probabilities [38,39].

\section{RESULTS}

We now apply the extended CCS methodology to explore the effect of tuning the Zeeman states of the reactants on the ultracold chemical reaction $\mathrm{Li}+\mathrm{CaH} \rightarrow \mathrm{LiH}+\mathrm{Ca}$. Figure 2 shows the collision energy dependence of the reaction cross section calculated for the different spin states of the reactants $\left|S_{i} m_{S_{i}}\right\rangle$ with the hyperfine structure omitted for the moment. The reaction cross section for spin-antialigned reactants $\left|\frac{1}{2},-\frac{1}{2}\right\rangle_{\mathrm{Li}}+\left|\frac{1}{2}, \frac{1}{2}\right\rangle_{\mathrm{CaH}}$ decreases with the collision energy $E_{C}$ as expected for the Langevin cross section $\left(\sigma^{R} \simeq\right.$ $\left.E^{-1 / 3}[60]\right)$. By averaging the dependence $\sigma^{R}(E)$ over a Maxwell-Boltzmann distribution of collision energies, we obtain the reaction rate in quantitative agreement with the 


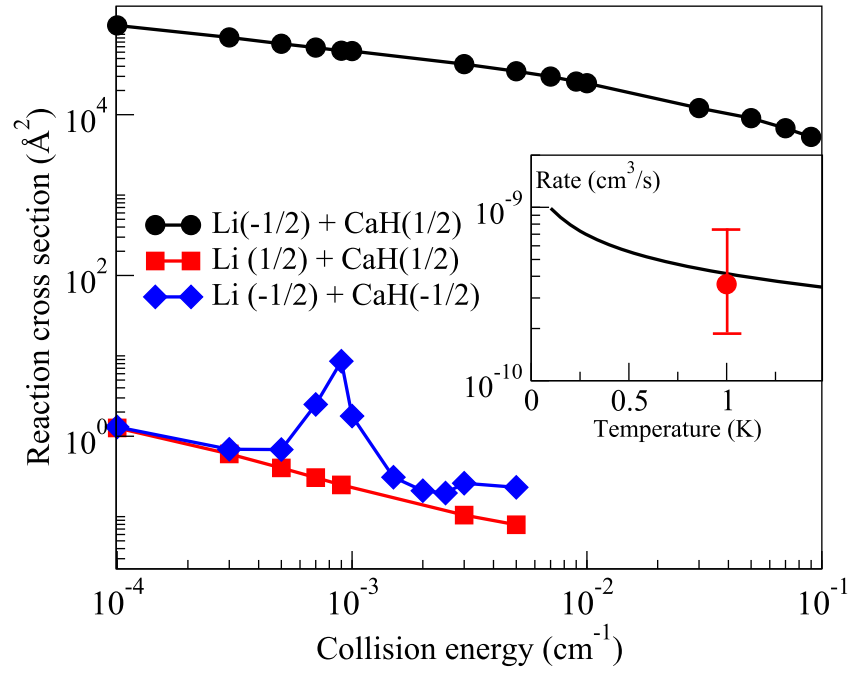

FIG. 2. Total reaction cross sections for $\left|\frac{1}{2}, \frac{1}{2}\right\rangle_{\mathrm{Li}}+\left|\frac{1}{2}, \frac{1}{2}\right\rangle_{\mathrm{CaH}}$ (squares) and $\left|\frac{1}{2},-\frac{1}{2}\right\rangle_{\mathrm{Li}}+\left|\frac{1}{2}, \frac{1}{2}\right\rangle_{\mathrm{CaH}}$ (circles) as a function of the collision energy at a magnetic field of $0.01 \mathrm{~T}$. Inset: $\mathrm{Li}+\mathrm{CaH}$ reaction rate plotted as a function of temperature (solid line) vs experimental result [54] (circle). The legends indicate the initial $m_{S}$ values of $\mathrm{Li}$ and $\mathrm{CaH}$.

measured value of $3.6 \times 10^{-10} \mathrm{~cm}^{3} / \mathrm{s}$ [54]. Because our CCS calculations assume unit probability of short-range loss for the reactive channels (see Appendix A), we conclude that nonuniversal effects are not significant for the spin-antialigned $\mathrm{Li}+\mathrm{CaH}$ reaction at $1 \mathrm{~K}$.

As shown in Fig. 2, the reaction of spin-aligned reactants $\left|\frac{1}{2}, \frac{1}{2}\right\rangle_{\mathrm{Li}}+\left|\frac{1}{2}, \frac{1}{2}\right\rangle_{\mathrm{CaH}}$ is suppressed by four orders of magnitude compared to that of spin-antialigned initial states. The spinaligned reaction nevertheless occurs through the intramolecular spin-rotation and intermolecular magnetic dipole-dipole interactions, which flip the total spin of the Li-CaH complex in the entrance reaction channel $[33,34]$. Because these interactions are weak, the spin-aligned reaction rate is small, and is comparable to that of nonreactive spin relaxation in $\left|\frac{1}{2}, \frac{1}{2}\right\rangle_{\mathrm{Li}}+$ $\left|\frac{1}{2}, \frac{1}{2}\right\rangle_{\mathrm{CaH}}$ collisions [56]. A resonance feature occurs at a collision energy of $\sim 1 \mathrm{mK}$ in the $\left|\frac{1}{2},-\frac{1}{2}\right\rangle_{\mathrm{Li}}+\left|\frac{1}{2},-\frac{1}{2}\right\rangle_{\mathrm{CaH}}$ initial channel, which could be tentatively assigned to a shape resonance corresponding to the orbital angular momentum $l=1$.

We next explore the effects of external magnetic fields and hyperfine interactions on chemical reactivity. Figure 3 shows the magnetic field dependence of reaction cross sections for the different initial hyperfine states of $\mathrm{Li}$ and $\mathrm{CaH}$ [see Figs. 1(b) and 1(c)]. We observe that certain combinations of initial hyperfine states are far more reactive than others: In particular, changing the initial state from $|2,2\rangle_{\mathrm{Li}}+|10\rangle_{\mathrm{CaH}}$ to $|2,-2\rangle_{\mathrm{Li}}+|11\rangle_{\mathrm{CaH}}$ enhances the reaction by an order of magnitude. This suggests the possibility of controlling ultracold reaction rates by tuning the hyperfine states of the reactants, which could be realized experimentally via radio-frequency and/or optical pumping.

Remarkably, as shown in Fig. 3, the reactivities of selected initial hyperfine states are extremely sensitive to the magnetic field strength, which opens up the prospect of controlling ultracold barrierless chemical reactions with external mag-

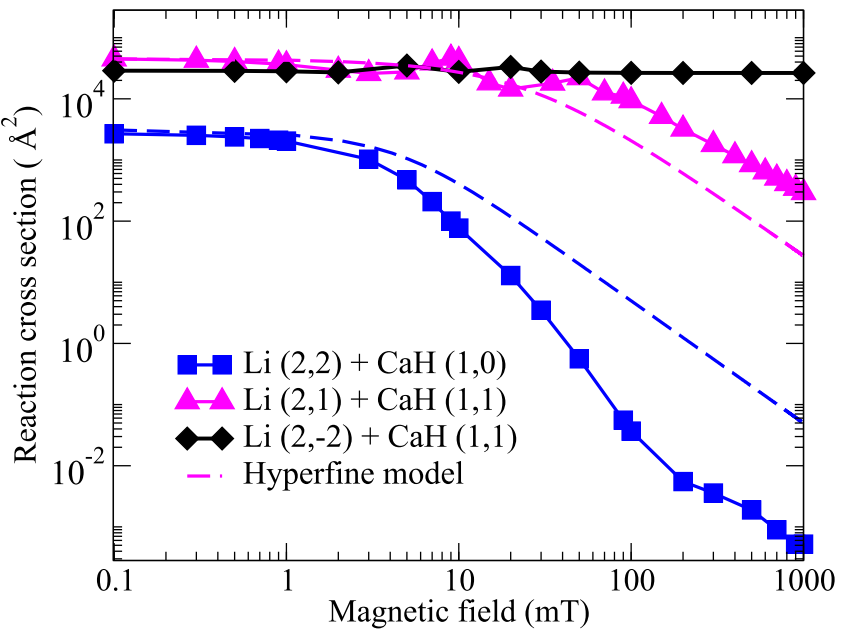

FIG. 3. (a) Total reaction sections for $\left|F m_{F}\right\rangle_{\mathrm{Li}}+\left|F^{\prime} m_{F}^{\prime}\right\rangle_{\mathrm{CaH}}$ as a function of the applied magnetic field. The collision energy is $10^{-3} \mathrm{~cm}^{-1}=1.4 \mathrm{mK}$. The dashed lines show the predictions of the hyperfine model.

netic fields. To gain insight into the field dependence of the reaction cross sections, we observe that the nuclear spin degrees of freedom (DOFs) do not directly participate in the reaction dynamics, which is governed instead by the electron spin DOFs. This implies [61,62] that the matrix elements of the atom-molecule PES are diagonal in the nuclear spin projections $m_{I_{A}}$ and $m_{I_{B}}$. The reaction cross sections are given in terms of the exact $S$-matrix elements

$$
\sigma_{\gamma_{A} m_{A} \gamma_{B} m_{B} \rightarrow f}^{R}=\frac{\pi}{k_{i}^{2}} \sum_{M}\left|S_{\gamma_{A} m_{A} \gamma_{B} m_{B} \rightarrow f}^{M}\right|^{2} .
$$

The initial Zeeman states $\left|\gamma_{i} m_{i}\right\rangle$ are linear combinations of the hyperfine states of atom $A$ and molecule $B(i=A, B)\left|\gamma_{i} m_{i}\right\rangle=$ $\sum_{m_{S_{i}}} C_{m_{S_{i}} m_{I_{i}}, \gamma_{i}}(B)\left|S_{i} m_{S_{i}}\right\rangle\left|I_{i} m_{I_{i}}\right\rangle$, with the $B$-dependent mixing coefficients $C_{m_{S_{i}} m_{I_{i}}, \gamma_{i}}(B)$ (suppressing the fixed labels $S_{i}$ ). Combining this with Eq. (2), we obtain

$$
\begin{aligned}
S_{\gamma_{A} m_{A} \gamma_{B} m_{B} \rightarrow f}^{M} & \sum_{m_{S_{A}}, m_{S_{B}}} C_{m_{S_{A}} m_{I_{A}}, \gamma_{A} m_{A}}(B) \\
& \times C_{m_{S_{B}} m_{I_{B}}, \gamma_{B} m_{B}}(B) S_{m_{S_{A}} m_{S_{B}} \rightarrow f}^{M} .
\end{aligned}
$$

We assume that the $S$-matrix elements on the right are independent of $B$ (which is approximately true as shown in Fig. 3) and they are different from zero only if $m_{S_{A}}$ and $m_{S_{B}}$ correspond to the reactive singlet state $(S=0)$ as discussed above. The magnetic field dependence of the reaction cross section is thus encapsulated in the hyperfine mixing coefficients $C_{m_{S_{i}} m_{I_{i}}, \gamma_{i} m_{i}}(B)$.

We now illustrate the hyperfine model (3) by applying it to the chemical reaction $|21\rangle_{\mathrm{Li}}+|11\rangle_{\mathrm{CaH}}$. From Eq. (3), we obtain the hyperfine $S$-matrix element as $S_{21,11 \rightarrow f}^{M}=$ $C_{-\frac{1}{2} \frac{3}{2}, 21}(B) S_{\frac{1}{2},-\frac{1}{2} \rightarrow f}^{M}$ and hence

$$
\sigma_{21,11 \rightarrow f}^{R}=\left|C_{-\frac{1}{2} \frac{3}{2}, 21}(B)\right|^{2} \sigma_{-\frac{1}{2}, \frac{1}{2} \rightarrow f}^{R},
$$

where $\sigma_{-\frac{1}{2}, \frac{1}{2} \rightarrow f}^{R}$ is the reaction cross section in the absence of the hyperfine structure (upper trace in Fig. 2). 
As shown in Fig. 3, the reaction cross sections predicted by the hyperfine model decrease as a function of the applied magnetic field, in qualitative agreement with the CCS results. The suppression is due to the decoupling of the electron and nuclear spins: As shown in Fig. 1(c), the initial hyperfine state $|2,1\rangle_{\mathrm{Li}}$ correlates with the nonreactive Zeeman state $\left|m_{S}=1 / 2\right\rangle_{\mathrm{Li}}$ in the high-field limit, leading to a decrease of the contribution of the reactive state $\left|m_{S}=-\frac{1}{2}\right\rangle_{\mathrm{Li}}$ with increasing field. The reaction cross section scales as $B^{-2}$ due to the mixing coefficient $C_{-\frac{1}{2}, \frac{3}{2}, 21}(B) \simeq B^{-1}$. We note that the hyperfine model predicts a less steep decline of the reaction cross sections with the field for the $|22\rangle_{\mathrm{Li}}+|10\rangle_{\mathrm{CaH}}$ initial state, whereas the opposite is true for $|21\rangle_{\mathrm{Li}}+|11\rangle_{\mathrm{CaH}}$. These deviations are expected because the hyperfine model neglects the effects of the magnetic field on the $S$-matrix elements, which are likely to become more pronounced at higher fields $[61,62]$.

\section{CONCLUSIONS AND OUTLOOK}

We have extended the rigorous CCS model of barrierless chemical reactions [38-40] to include the hyperfine structure of open-shell reactants and their interactions with external magnetic fields. We have applied the model to explore the effects of hyperfine interactions and magnetic fields on the dynamics of the prototypical barrierless chemical reaction $\mathrm{Li}+\mathrm{CaH} \rightarrow \mathrm{LiH}+\mathrm{Ca}$. Our calculated reaction rates agree with experiment [54] and display a dramatic dependence on the external magnetic field, which could be used to facilitate sympathetic cooling of chemically reactive ${ }^{2} \Sigma$ molecules with alkali-metal atoms [56,63]. We expect our approach to be readily applicable to a wide range of ultracold barrierless chemical reactions of current experimental interest, including those involving molecular ions [64-67] and alkaline-earth halides $\mathrm{SrF}$ and $\mathrm{CaF}[30,58,68]$. In future work, we intend to explore the connection between the extended CCS model and the universal model of chemical reactions [44-49], which would allow for the study of nonuniversal effects [46,69].

\section{ACKNOWLEDGMENTS}

We are grateful to David Manolopoulos, Gerrit Groenenboom, Paul Brumer, Roman Krems, John Bohn, and Masato Morita for discussions. This work was supported by NSF Grants No. PHY-1607610, No. PHY-1912668, and partially by the U.S. Air Force Office for Scientific Research (AFOSR) under Contract No. FA9550-19-1-0312. J.K. acknowledges financial support under NSF Grant No. CHE-156872 to M. H. Alexander.

\section{APPENDIX A: THE EXTENDED CCS MODEL: OVERVIEW AND NUMERICAL IMPLEMENTATION}

This Appendix provides an overview of the extended CCS approach. We begin by introducing the CC equations in Appendix A 1 and describing the procedure of applying the boundary conditions in Appendix A 2. Appendix A 3 describes further technical details pertaining to the evaluation of the matrix elements of the atom-molecule interaction and of the orbital angular momentum of the collision complex.

\section{Numerical solution of $\mathrm{CC}$ equations: Reaction cross sections and capture probabilities}

The CCS capture probability in the entrance reaction channel is given by [38-40]

$$
p_{\gamma_{A} \gamma_{B} l}^{M}=1-\sum_{\gamma_{A}^{\prime} \gamma_{B}^{\prime} l^{\prime}}\left|S_{\gamma_{A}^{\prime} \gamma_{B}^{\prime} l^{\prime}, \gamma_{A} \gamma_{B} l}^{M}\right|^{2},
$$

where $\gamma_{A}, \gamma_{B}$ and $\gamma_{A}^{\prime}, \gamma_{B}^{\prime}$ stand for the initial and final Zeeman states of the reactants, $l$ and $l^{\prime}$ are the corresponding orbital angular momenta, and $M$ is the space-fixed (SF) projection of the total angular momentum $J$ of the collision complex on the magnetic field axis, which is conserved for reactions in magnetic fields. The total reaction cross section is obtained by summing the entrance channel capture probabilities (A1) over a range of orbital angular momenta $l$ and total angular momentum projections $M$,

$$
\sigma_{\gamma_{A} \gamma_{B} \rightarrow f}=\frac{\pi}{k_{\gamma_{A} \gamma_{B}}^{2}} \sum_{M} \sum_{l} p_{\gamma_{A} \gamma_{B} l}^{M},
$$

where $k_{\gamma_{A} \gamma_{B}}=2 \mu E_{C}$ is the wave vector in the incident collision channel and $E_{C}$ is the collision energy. We note that the reaction cross section can be obtained from the fully stateto-state cross section by summing over the final $\mathrm{LiH}+\mathrm{Ca}$ product states $\gamma_{A}^{\prime}$ and $\gamma_{B}^{\prime}$.

The $S$-matrix elements in Eq. (A1) are obtained from the radial solutions $F_{\alpha_{A} \alpha_{B} J \Omega}^{M}(R)$ of the coupled-channel (CC) equations at total energy $E$ [38-40],

$$
\begin{aligned}
& {\left[\frac{d^{2}}{d R^{2}}+2 \mu E\right] F_{\alpha_{A} \alpha_{B} J \Omega}^{M}(R)} \\
& \quad=2 \mu \sum_{\alpha_{A}^{\prime} \alpha_{B}^{\prime}} \sum_{J^{\prime}, \Omega^{\prime}}\left\langle\alpha_{A} \alpha_{B}\right|\langle J M \Omega| \hat{V}(R, r, \theta)+\frac{\hat{L}^{2}}{2 \mu R^{2}} \\
& \quad+\hat{V}(R, r, \theta)+\hat{H}_{\mathrm{as}}\left|\alpha_{A}^{\prime} \alpha_{B}^{\prime}\right\rangle\left|J^{\prime} M \Omega^{\prime}\right\rangle F_{\alpha_{A}^{\prime} \alpha_{B}^{\prime} J^{\prime} \Omega^{\prime}}^{M}(R),
\end{aligned}
$$

subject to the capture boundary conditions as described below. The CC equations (A3) describe atom-molecule scattering in the entrance reaction channel in the presence of an external magnetic field. In Eq. (A3)

$$
\begin{aligned}
\left|\alpha_{A} \alpha_{B}\right\rangle|J M \Omega\rangle= & \left|N_{A} K_{N_{A}}\right\rangle\left|S_{A} \Sigma_{A}\right\rangle\left|I_{A} \Sigma_{I_{A}}\right\rangle \\
& \times\left|S_{B} \Sigma_{B}\right\rangle\left|I_{B} \Sigma_{I_{B}}\right\rangle|J M \Omega\rangle
\end{aligned}
$$

are body-fixed (BF) basis functions for the overall rotational motion $(|J M \Omega\rangle)$ and the internal degrees of freedom of molecule $A\left(\alpha_{A}\right)$ and atom $B\left(\alpha_{B}\right)$, including the rotational angular momentum $N_{A}$, the electron spins $\hat{S}_{A}$ and $\hat{S}_{B}$, and the nuclear spins $\hat{I}_{A}$ and $\hat{I}_{B}$, with $K_{N_{A}}, \Sigma_{A}, \Sigma_{B}, \Sigma_{I_{A}}$, and $\Sigma_{I_{B}}$ being the projections of $N_{A}, S_{A}, S_{B}, I_{A}$, and $I_{B}$ on the atom-diatom separation vector $\boldsymbol{R}$ chosen as the $z$ axis of the BF coordinate frame [59]. The matrix elements are evaluated as described in Appendix A 3 below.

To validate the extended CCS model of atom-molecule insertion reactions in a magnetic field, we have also formulated and solved $\mathrm{CC}$ equations in the fully uncoupled space-fixed (SF) representation [70,71]

$$
\left|N_{A} M_{N_{A}}\right\rangle\left|S_{A} m_{S_{A}}\right\rangle\left|I_{A} m_{I_{A}}\right\rangle\left|S_{B} m_{S_{B}}\right\rangle\left|I_{B} m_{I_{B}}\right\rangle\left|l m_{l}\right\rangle,
$$


where we have defined the projections of the rotational angular momentum $\left(M_{N_{A}}\right)$, electron spin $\left(m_{S_{A}}\right)$, and nuclear spin $\left(m_{I_{A}}\right)$ of molecule $A$ on the magnetic field axis, along with the corresponding projections for atom $B\left(m_{S_{B}}\right.$ and $\left.m_{I_{B}}\right)$. The projection of the orbital angular momentum of the collision complex $l$ on the field axis is denoted by $m_{l}$. The matrix elements of the asymptotic Hamiltonian, the interaction potential, and the centrifugal kinetic energy are evaluated as described in our previous work [72].

Unlike the BF basis states (A4), the SF basis states (A5) do not have a well-defined value of the total angular momentum $J$. As a result, reaching numerical convergence in the SF representation generally requires much larger rotational basis sets [59]. As shown below, CCS calculations produce converged results already for small rotational basis sets $\left(N_{\max }=2\right)$, so the use of the SF basis is justified.

Test calculations show that both the SF and BF formulations give identical results for the $\mathrm{Li}+\mathrm{CaH}$ chemical reaction in the absence of the hyperfine interaction, thereby validating the extended CCS model. However, we found that BF calculations in the presence of hyperfine interactions suffer from numerical instabilities, which could arise due to the presence of unphysical Zeeman states [59]. As the SF formulation of the CCS theory is free from the unphysical states, we use it to calculate the reaction cross sections in the presence of hyperfine interactions (shown in Fig. 3 of the main text).

\section{Boundary conditions}

We solve the CC equations numerically by constructing the $\log$-derivative matrix $\mathbf{Y}=\boldsymbol{\Psi}^{-1} \boldsymbol{\Psi}$, where $\Psi$ is the wave function matrix, and propagating it from a small value of $R=$ $R_{c}$ out to the asymptotic region [38-40]. We choose an initial value of the capture radius $R=R_{c}$ inside the reaction complex region and initialize the complex symmetric log-derivative matrix as [38-40]

$$
\mathbf{Y}\left(R_{c}\right)=\mathbf{C}\left(R_{c}\right) \mathbf{Y}_{d}\left(R_{c}\right) \mathbf{C}^{T}\left(R_{c}\right),
$$

where $\mathbf{Y}_{d}\left(R_{c}\right)=\left(y_{1}\left(R_{c}\right), y_{2}\left(R_{c}\right), \ldots, y_{N}\left(R_{c}\right)\right)$ is the diagonal matrix constructed from the eigenvalues $\epsilon_{c}=-k_{c}^{2}$ of the coupling matrix $\mathbf{W}\left(R_{c}\right)$,

$$
\begin{aligned}
{\left[\mathbf{W}\left(R_{c}\right)\right]_{n n^{\prime}}=} & \langle J M \Omega|\left\langle\alpha_{A} \alpha_{B}\right| V\left(R_{c}, r, \theta\right)+\frac{L^{2}}{2 \mu R_{c}^{2}} \\
& +\hat{H}_{\mathrm{as}}\left|\alpha_{A}^{\prime} \alpha_{B}^{\prime}\right\rangle\left|J^{\prime} M^{\prime} \Omega^{\prime}\right\rangle,
\end{aligned}
$$

using the multichannel Wentzel-Kramers-Brillouin (WKB) boundary conditions [40]. The entrance channels of chemical reactions that occur on multiple PESs (such as the Li$\mathrm{CaH}$ reaction considered here) typically include the highly attractive as well as strongly repulsive PESs, leading to two qualitatively different types of adiabatic channels illustrated in Fig. 4. The reactive channels decrease in energy with decreasing $R<R_{c}$, whereas the nonreactive channels show the opposite trend. Both types of channels can be treated on an equal footing using the Airy boundary conditions $[40,73]$. Following Ref. [73], we initialize the elements of the diagonal matrix $\mathbf{Y}_{d}$ as

$$
y\left(R_{c}\right)=W^{\prime}\left(R_{c}\right)^{1 / 3} \frac{\phi^{\prime}\left(x_{c}\right)}{\phi\left(x_{c}\right)},
$$

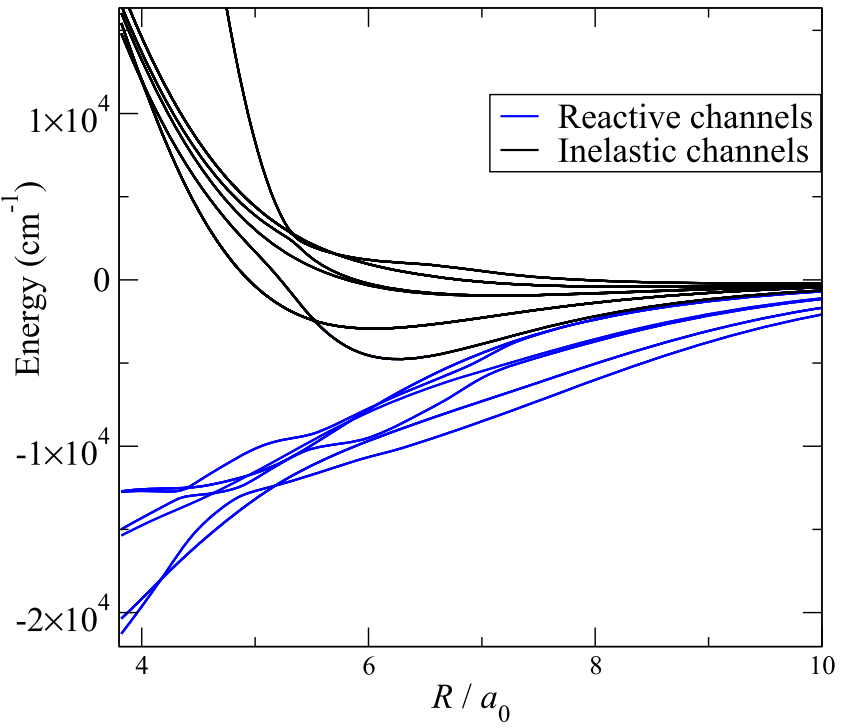

FIG. 4. Reactive [blue (gray) lines] and inelastic (black lines) adiabatic channels calculated for $\mathrm{Li}-\mathrm{CaH}$ using a restricted basis set with $N_{\max }=2$ and total angular momentum projection $M=0$ at a magnetic field of $0.01 \mathrm{~T}$. Note the different asymptotic behavior of the inelastic and reactive channels at short $R$.

where $W^{\prime}\left(R_{c}\right)^{1 / 3}$ is the real root of $x^{3}=W^{\prime}\left(R_{c}\right)$, and $W^{\prime}\left(R_{c}\right)$ is the derivative of the adiabatic eigenvalue $W\left(R_{c}\right)$ (an eigenvalue of matrix $\mathbf{W}$ ), which is positive (negative) for reactive (inelastic) channels (see Fig. 4). The scaled wave function $\phi(x)$ is a solution of the Airy equation for the adiabatic channels linearly extrapolated into the reaction complex region $R<R_{c}[$ [73]

$$
\phi^{\prime \prime}(x)=\frac{a^{3}}{W^{\prime}\left(R_{c}\right)^{2}} x \phi(x),
$$

where $x=W(R) /\left[W^{\prime}(R)^{2 / 3}\right]$ is a scaled radial coordinate.

For $W^{\prime}\left(R_{c}\right)>0$ (reactive channels) and $R \ll R_{c}$, we have $x \rightarrow-\infty$ and the wave function ratio in Eq. (A8) takes the form [73]

$$
\frac{\phi^{\prime}(x)}{\phi(x)}=\frac{\mathrm{Bi}^{\prime}(x)+i \mathrm{Ai}^{\prime}(x)}{\mathrm{Bi}(x)+i \operatorname{Ai}(x)} \stackrel{x \rightarrow-\infty}{\longrightarrow}-i \sqrt{-x}-\frac{1}{4 x},
$$

where $\operatorname{Bi}(x)$ and $\operatorname{Ai}(x)$ are the Airy functions, which oscillate in the limit of large negative $x$.

For $W^{\prime}\left(R_{c}\right)<0$ (inelastic channels) and $R \ll R_{c}$, we have $x \rightarrow+\infty$. Retaining only the asymptotically decaying Airy function $\operatorname{Ai}(x)$, we obtain the wave function ratio in Eq. (A8) as [73]

$$
\frac{\phi^{\prime}(x)}{\phi(x)}=\frac{\operatorname{Ai}^{\prime}(x)}{\operatorname{Ai}(x)} \stackrel{x \rightarrow+\infty}{\longrightarrow} \sqrt{x}-\frac{1}{4 x} .
$$

In practice, the asymptotic expressions (A10) and (A11) give sufficiently accurate results for $|x| \geqslant 5$. However, in our numerical calculations a small fraction of adiabatic channels has $|x|<5$, making it necessary to apply numerically exact expressions for the Airy functions.

At a large atom-molecule distance $R=R_{a}$, we match the log-derivative matrix $\mathbf{Y}\left(R_{a}\right)$ to the standard incoming and outgoing wave boundary conditions to obtain the scattering 
$S$ matrix $[38,39]$

$$
\mathbf{S}=\left[\mathbf{Y}\left(R_{a}\right) \mathbf{O}_{E}\left(R_{a}\right)-\mathbf{O}_{E}^{\prime}\left(R_{a}\right)\right]^{-1}\left[\mathbf{Y}\left(R_{a}\right) \mathbf{I}_{E}\left(R_{a}\right)-\mathbf{I}_{E}^{\prime}\left(R_{a}\right)\right],
$$

where $\mathbf{I}_{E}$ and $\mathbf{O}_{E}$ are the diagonal matrices composed of the incoming- and outgoing-wave solutions of CC equations in the absence of the atom-molecule interaction (for open channels)

$$
\begin{aligned}
{\left[I_{E}\left(R_{a}\right)\right]_{\gamma l, \gamma^{\prime} l^{\prime}} } & =\delta_{\gamma \gamma^{\prime}} \delta_{l l^{\prime}} k_{\gamma}^{1 / 2} R h_{l}^{(2)}\left(k_{\gamma} R\right), \\
{\left[O_{E}\left(R_{a}\right)\right]_{\gamma l, \gamma^{\prime} l^{\prime}} } & =\delta_{\gamma \gamma^{\prime}} \delta_{l l^{\prime}} k_{\gamma}^{1 / 2} R h_{l}^{(1)}\left(k_{\gamma} R\right),
\end{aligned}
$$

where $\gamma$ is a compound index for $\gamma_{A}, \gamma_{B}, k_{\gamma}=\left[2 \mu E_{C}\right]^{1 / 2}$ is the incident wave vector, $E_{C}$ is the collision energy, and $h_{l}^{( \pm)}(x)$ are the spherical Hankel functions. The asymptotic solutions for closed channels are given by [39]

$$
\begin{aligned}
{\left[I_{E}\left(R_{a}\right)\right]_{\gamma l, \gamma^{\prime} l^{\prime}} } & =\delta_{\gamma \gamma^{\prime}} \delta_{l l^{\prime}}\left|k_{\gamma}\right|^{1 / 2} R i_{l}\left(\left|k_{\gamma}\right| R\right), \\
{\left[O_{E}\left(R_{a}\right)\right]_{\gamma l, \gamma^{\prime} l^{\prime}} } & =\delta_{\gamma \gamma^{\prime}} \delta_{l l^{\prime}}\left|k_{\gamma}\right|^{1 / 2} R k_{l}\left(\left|k_{\gamma}\right| R\right),
\end{aligned}
$$

where $i_{l}(x)$ and $k_{l}(x)$ are the modified spherical Bessel functions.

\section{Matrix elements}

We now turn to the evaluation of the matrix elements in Eq. (A3). The asymptotic Hamiltonian may be written as [59]

$$
\hat{H}_{\mathrm{as}}=\hat{H}_{A}+\hat{H}_{B},
$$

where $\hat{H}_{A}$ and $\hat{H}_{B}$ are the asymptotic Hamiltonians of the reactants [molecule $A\left({ }^{2} \Sigma\right)$ plus atom $B\left({ }^{2} S\right)$ ]. The molecular Hamiltonian is given by $[72,74]$

$$
\begin{aligned}
\hat{H}_{A}= & B_{e} \boldsymbol{N}_{A}^{2}+\gamma \hat{\boldsymbol{N}}_{A} \cdot \hat{\boldsymbol{S}}_{A}+(b+c / 3) \hat{\boldsymbol{I}}_{A} \cdot \hat{\boldsymbol{S}}_{A}+\frac{c \sqrt{6}}{3}\left(\frac{4 \pi}{5}\right)^{1 / 2} \\
& \times \sum_{q=-2}^{2}(-1)^{q} Y_{2-q}\left(\hat{r}_{A}\right)\left[\hat{\boldsymbol{I}}_{A} \otimes \hat{\boldsymbol{S}}_{A}\right]_{q}^{(2)}+2 \mu_{0} B S_{A_{Z}}, \quad \text { (A16) }
\end{aligned}
$$

where $\hat{\boldsymbol{N}}_{A}$ is the rotational angular momentum, $\hat{\boldsymbol{S}}_{A}$ and $\hat{\boldsymbol{I}}_{A}$ are the electron and nuclear spins with space-fixed (SF) projections $\hat{S}_{Z_{A}}$ and $\hat{I}_{Z_{A}}\left[I_{A}=S_{A}=1 / 2\right.$ for $\left.\mathrm{CaH}\left(X^{2} \Sigma\right)\right], \hat{\boldsymbol{I}}_{A} \otimes \hat{\boldsymbol{S}}_{A}$ is a tensor product of $\hat{\boldsymbol{I}}_{A}$ and $\hat{\boldsymbol{S}}_{A}, Y_{2-q}(\hat{r})$ is a spherical harmonic describing the orientation of the molecular axis $\hat{r}_{A}$ in the SF frame, and $B_{e}, \gamma, b$, and $c$ are the rotational, spin-rotation, and hyperfine constants. We neglect the weak nuclear spinrotation interaction [72]. The atomic Hamiltonian

$$
\hat{H}_{B}=A_{B} \hat{\boldsymbol{I}}_{B} \cdot \hat{\boldsymbol{S}}_{B}+2 \mu_{0} B S_{B_{Z}}
$$

includes the hyperfine coupling of the electron and nuclear spins parametrized by the atomic hyperfine constant $A_{B}$, and the interaction of the atomic spin with an external magnetic field $B$. Writing the asymptotic Hamiltonian (A15) as a sum of field-free and Zeeman terms

$$
\hat{H}_{\mathrm{as}}=\hat{H}_{A}^{(0)}+\hat{H}_{Z, A}+\hat{H}_{B}^{(0)}+\hat{H}_{Z, B},
$$

and taking advantage of the direct-product structure of the BF basis set (A4), we obtain

$$
\begin{aligned}
& \left\langle\alpha_{A} \alpha_{B}\left|\left\langle J M \Omega\left|\hat{H}_{\mathrm{as}}\right| \alpha_{A}^{\prime} \alpha_{B}^{\prime}\right\rangle\right| J^{\prime} M \Omega^{\prime}\right\rangle \\
& =\delta_{J J^{\prime}} \delta_{\Omega \Omega^{\prime}}\left[\delta_{\alpha_{B} \alpha_{B}^{\prime}}\left\langle\alpha_{A}\left|\hat{H}_{A}^{(0)}\right| \alpha_{A}^{\prime}\right\rangle+\delta_{\alpha_{A} \alpha_{A}^{\prime}}\left\langle\alpha_{B}\left|\hat{H}_{B}^{(0)}\right| \alpha_{B}^{\prime}\right\rangle\right] \\
& \quad+\delta_{\alpha_{B} \alpha_{B}^{\prime}}\left\langle\alpha_{A}\left|\left\langle J M \Omega\left|\hat{H}_{Z, A}\right| \alpha_{A}^{\prime}\right\rangle\right| J^{\prime} M \Omega^{\prime}\right\rangle \\
& \quad+\delta_{\alpha_{A} \alpha_{A}^{\prime}}\left\langle\alpha_{B}\left|\left\langle J M \Omega\left|\hat{H}_{Z, B}\right| \alpha_{B}^{\prime}\right\rangle\right| J^{\prime} M \Omega^{\prime}\right\rangle .
\end{aligned}
$$

The matrix elements on the right can be evaluated as described in our previous work $[59,72]$.

To calculate the matrix elements of the orbital angular momentum operator in Eq. (A3) in the body-fixed angular momentum basis, we express the operator in the form

$$
\hat{L}^{2}=\left(\hat{J}-\hat{N}_{A}-\hat{S}_{A}-\hat{S}_{B}-\hat{I}_{A}-\hat{I}_{B}\right)^{2} .
$$

While $\hat{L}^{2}$ can be expressed in terms of the raising and lowering operators for all angular momenta involved as done, e.g., in Ref. [59], the resulting expressions are rather cumbersome due to the presence of two additional nuclear spin operators $\hat{I}_{A}$ and $\hat{I}_{B}$. To simplify the evaluation of the angular momentum matrix elements, it is convenient to define the orbital angular momentum of the atom-molecule system in the absence of the nuclear spin,

$$
\hat{L}_{4}=\hat{J}-\hat{N}_{A}-\hat{S}_{A}-\hat{S}_{B} .
$$

The matrix elements of this operator can be evaluated as described in our previous work [59]. To incorporate the nuclear spins, we combine Eqs. (A20) and (A21) and use the fact that the nuclear spin operators commute with $\hat{L}_{4}$ to obtain

$$
\begin{aligned}
\hat{L}^{2} & =\left(\hat{L}_{4}-\hat{I}_{A}-\hat{I}_{B}\right)^{2} \\
& =\hat{L}_{4}^{2}+\hat{I}_{A}^{2}+\hat{I}_{B}^{2}-2 \hat{L}_{4} \cdot \hat{I}_{A}-2 \hat{L}_{4} \cdot \hat{I}_{B}+2 \hat{I}_{A} \cdot \hat{I}_{B} .
\end{aligned}
$$

Expressing the scalar products of angular momentum operators via the raising and lowering operators, e.g., $\hat{L}_{4} \cdot \hat{I}_{A}=\hat{L}_{4 z}$. $\hat{I}_{A z}+\frac{1}{2}\left(\hat{L}_{4+} \cdot \hat{I}_{A-}+\hat{L}_{4-} \cdot \hat{I}_{A+}\right)$, the evaluation of the matrix elements of the operator $\hat{L}^{2}$ (A20) in the basis (A4) reduces to straightforward angular momentum algebra described, e.g., in Ref. [59].

\section{APPENDIX B: AB INITIO CALCULATIONS AND PES FITTING}

To compute the singlet $(S=0)$ and triplet $(S=1)$ PESs of the $\mathrm{Li}-\mathrm{CaH}$ reaction complex, we used high-level multireference configuration interaction (MRCI) and coupledcluster methods with single, double, and noniterative triple excitations as implemented in the MOLPRO code [75].

The triplet PES is calculated as described in our previous work [56] at the CCSD(T) level of theory [76]. To compute the singlet $(S=0)$ PES, we took into account the multireference character of the electronic wave function by using the MRCI method [77] with single and double excitations (MRCISD) and Davidson corrections $(+Q)$ to approximately account for contributions of higher excitations. The MRCISD + Q calculations were started from the reference orbitals obtained at the state-averaged complete active space self-consistent-field (sa-CASSCF) level treating all $S$ states on the same footing. The active space contained $4 A^{\prime}$ and $1 A^{\prime \prime}$ orbitals and 6 orbitals 
were correlated but kept doubly occupied $\left(5 A^{\prime}\right.$ and $\left.1 A^{\prime \prime}\right)$. The frozen core of the $\mathrm{Ca}$ atom was composed of the $4 A^{\prime}$ and $1 A^{\prime \prime}$ orbitals. We used the augmented, correlation consistent triplezeta (aug-cc-pvtz) basis for $\mathrm{H}$ [78], a quadruple-zeta basis (aug-cc-pvqz) for $\mathrm{Li}$, and a valence quadruple-zeta (cc-pvqz) basis for $\mathrm{Ca}$ [79].

The $a b$ initio calculations were performed on a twodimensional grid of $R$ and $\theta$, with $\theta \in 0^{\circ}-180^{\circ}$ in steps of $5^{\circ}$ and $R \in 3.5-30 a_{0}$ [75]. To facilitate the calculation of the matrix elements of the atom-molecule interaction operator $\hat{V}(R, r, \theta)=\sum_{S M_{S}} V_{S}(R, \theta, r)\left|S M_{S}\right\rangle\left\langle S M_{S}\right|$, where $S$ is the total spin of the reaction complex, we expand the adiabatic potential energy surfaces $V_{S}(R, \theta, r)$ in Legendre polynomials $P_{\lambda}(\cos \theta)$

$$
V_{S}(R, r, \theta)=\sum_{\lambda=0}^{\lambda_{\max }} V_{\lambda}^{S}(R, r) P_{\lambda}(\cos \theta)
$$

Because we neglect the weak nuclear spin-dependent interactions that depend on $R$ (such as the interaction of the nuclear spins with the overall rotation of the reaction complex), the matrix elements of the interaction potential are diagonal in the nuclear spin quantum numbers $\Sigma_{I_{A}}$ and $\Sigma_{I_{B}}$ [see Eq. (A4)]. As a result, the matrix elements of Eq. (B1) are given by the expressions similar to Eqs. (30) and (31) of Ref. [59].

For use in quantum scattering calculations, the ab initio data points are expanded in Legendre polynomials (B1) with $\lambda_{\max }=18$ (for $S=0$ ) and $\lambda_{\max }=14$ (for $S=1$ ). The resulting radial expansion coefficients $V_{\lambda}(R)$ are fitted using the reproducing kernel Hilbert space method of Rabitz and coworkers [80]. To avoid unphysical distortion of the fit, we damped the very high repulsive energies at small $R$.

Following our previous work [53], we invoke the rigidrotor approximation by freezing the internuclear distance of $\mathrm{CaH}$ at its equilibrium value $r_{e}=2.0025 \AA$. This approximation provides quantitatively accurate capture probabilities for the $\mathrm{Li}-\mathrm{CaH}$ chemical reaction on a single adiabatic PES [53] at a much reduced computational cost. However, in the context of the CCS model, the rigid-rotor approximation leads to all adiabatic potentials becoming repulsive (i.e., nonreactive) at sufficiently short $R$. To address this, we introduce the following modification of the isotropic part of the singlet (reactive) PES

$$
\begin{aligned}
V_{0}^{0}(R)= & V_{0}^{0}\left(R_{c}\right)+\left.\frac{d V_{0}^{0}(R)}{d R}\right|_{R_{m}}\left(R-R_{m}\right) \\
& +\left.\frac{1}{2} \frac{d^{2} V_{0}^{0}(R)}{d R^{2}}\right|_{R_{m}}\left(R-R_{m}\right)^{2} \quad\left(R<R_{m}\right),
\end{aligned}
$$

where $R_{m}$ is a matching point to the right of the potential minimum, where the first and second derivatives of the potential have opposite signs with the second derivative being negative, so as to ensure the decreasing behavior of Eq. (B2) with decreasing $R<R_{m}$ [see Fig. 5(a)]. The modification replaces the short-range repulsive wall of the rigid-rotor potential with a function that decreases with $R$. This results in a one-parameter family of modified potentials parameterized by the values of $R_{m}$. We have verified [see Fig. 5(b)] that the calculated capture probabilities are insensitive to the choice of $R_{m}$ in the range of $R_{m}=7.0-7.7 a_{0}$. At $R_{m}<7 a_{0}$ the slope of
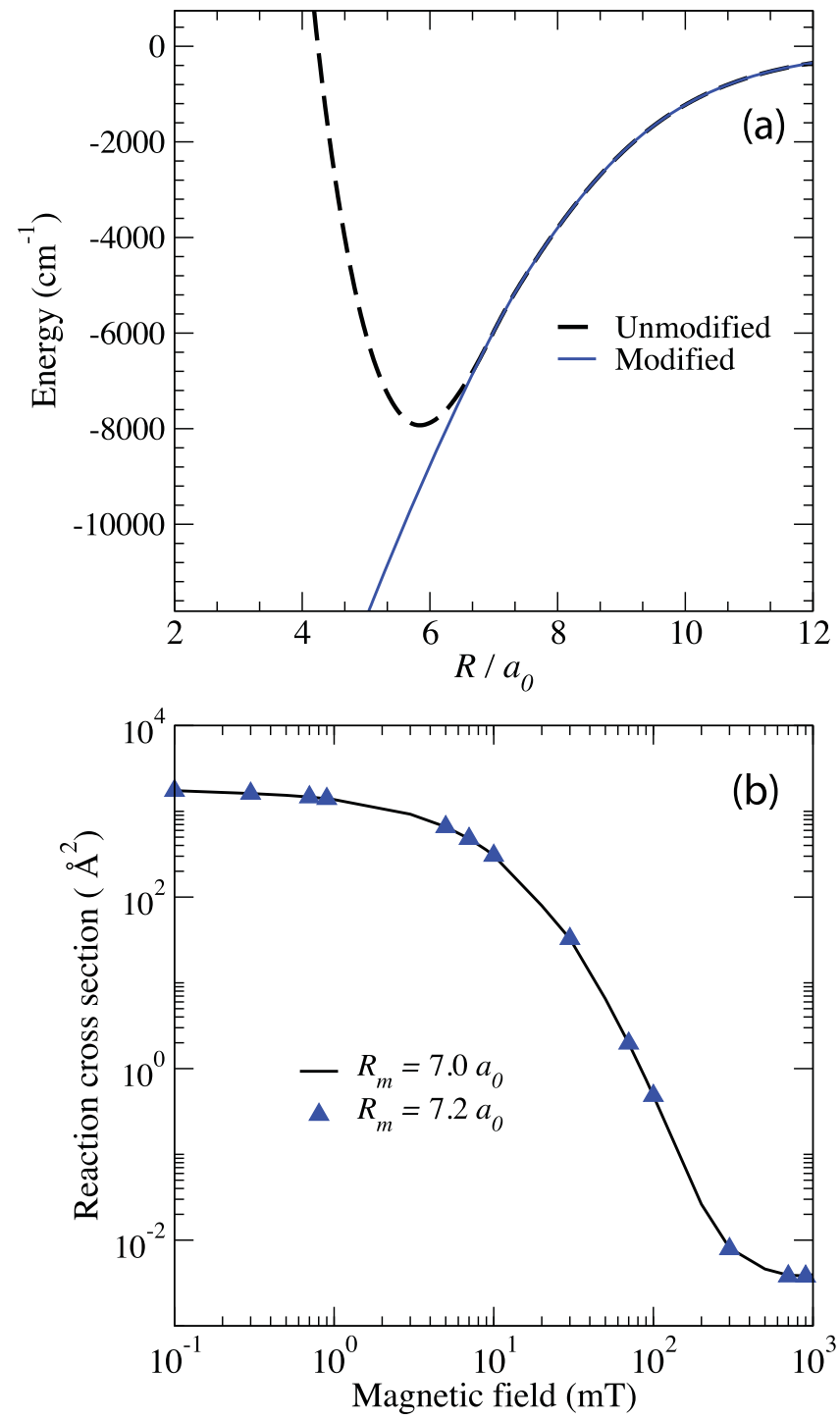

FIG. 5. (a) Unmodified (dashed line) and modified (full line) isotropic parts of the singlet Li-CaH PES in the rigid-rotor approximation $V_{0}^{S=0}(R)$. The value of the matching distance $R_{m}=$ $7 a_{0}$. (b) Magnetic field dependence of the reaction cross section calculated for several values of the matching parameter $R_{m}$ (in units of $a_{0}$ ) and the reactants' initial state $|21\rangle_{\mathrm{Li}}+|1,1\rangle_{\mathrm{CaH}}$ at a collision energy of $0.001 \mathrm{~cm}^{-1}$.

the modified potential [Fig. 5(a)] decreases toward zero near the minimum of the unmodified potential at $R=6 a_{0}$. As a result, the modified potential does not fall down fast enough with decreasing $R$ to ensure the proper short-range behavior of the reactive channels illustrated in Fig. 4, and we observe a significant artifactual change of the calculated reaction cross sections. We therefore used $R_{m}=7.1 a_{0}$ in all calculations.

\section{APPENDIX C: CONVERGENCE TESTS}

We carried out a series of convergence tests to determine the optimal values of the radial grid parameters $R_{c}$ (capture radius), $R_{a}$ (asymptotic matching distance), and $\Delta R$ (radial grid step), as well as the cutoff parameters $N_{\max }$ and $J_{\max }$ 
that determine the sizes of the rotational and total angular momentum basis sets. The same value of the capture radius $R_{c}=3.84 a_{0}$ was used in all calculations. Test calculations show that varying $R_{c}$ within the modified region of the potential $\left(R_{c}=3-5 a_{0}\right)$ changes the capture cross sections by less than $2 \%$. We use the following values of $R_{a}$ to obtain the capture probabilities and reaction cross sections converged to within 10\%-20\%: $R_{a}=360 a_{0}\left(E_{C}=\right.$ $10^{-4}$ to $\left.10^{-3} \mathrm{~cm}^{-1}\right), R_{a}=270 a_{0}\left(E_{C}=10^{-3}\right.$ to $\left.10^{-2} \mathrm{~cm}^{-1}\right)$, and $R_{a}=200 a_{0}\left(E_{C}>10^{-2} \mathrm{~cm}^{-1}\right)$, with a uniform grid step of $0.02 a_{0}$.

At the lowest collision energies studied in this work $\left(10^{-4}<E_{C}<10^{-3} \mathrm{~cm}^{-1}\right)$ it is sufficient to truncate the total angular momentum basis at $J_{\max }=2$ to produce results converged to $<5 \%$. At higher collision energies, progressively higher values of $J_{\max }$ were used, up to $J_{\max }=10$ at $E_{C}=$ $7 \mathrm{~cm}^{-1}$.

We also carried out convergence tests with respect to the maximum number of rotational states $N_{\max }$ included in the basis set. The capture cross sections for the spin-antialigned initial states $\left|\frac{1}{2},-\frac{1}{2}\right\rangle_{\mathrm{Li}}+\left|\frac{1}{2}, \frac{1}{2}\right\rangle_{\mathrm{CaH}}$ are large and remarkably insensitive to $N_{\max }$ as shown in Fig. 6. This suggests that anisotropic effects in the entrance channel of the $\mathrm{Li}+\mathrm{CaH}$ reaction play a minor role. For these initial states, a minimal basis set with $N_{\max }=2$ was used. In contrast, the small capture probabilities of spin-aligned reactants tend to be highly sensitive to the value of $N_{\max }$, making it necessary to employ much larger rotational basis sets with $N_{\max }=55$.

[1] R. N. Zare, Science 279, 1875 (1998).

[2] M. Shapiro and P. Brumer, Quantum Control of Molecular Processes (Wiley-VCH, Weinheim, 2012).

[3] D. Herschbach, Eur. Phys. J. D 38, 3 (2006).

[4] W. E. Perreault, N. Mukherjee, and R. N. Zare, Science 358, 356 (2017).

[5] W. E. Perreault, N. Mukherjee, and R. N. Zare, Nat. Chem. 10, 561 (2018).

[6] R. V. Krems, Phys. Chem. Chem. Phys. 10, 4079 (2008).

[7] N. Balakrishnan, J. Chem. Phys. 145, 150901 (2016).

[8] J. L. Bohn, A. M. Rey, and J. Ye, Science 357, 1002 (2017).

[9] M. Lemeshko, R. V. Krems, J. M. Doyle, and S. Kais, Mol. Phys. 111, 1648 (2013).

[10] A. Klein, Y. Shagam, W. Skomorowski, P. S. Żuchowski, M. Pawlak, L. M. C. Janssen, N. Moiseyev, S. Y. T. van de Meerakker, A. van der Avoird, C. P. Koch et al., Nat. Phys. 13, 35 (2016).

[11] N. Balakrishnan and A. Dalgarno, Chem. Phys. Lett. 341, 652 (2001).

[12] J. W. Park, Z. Z. Yan, H. Loh, S. A. Will, and M. W. Zwierlein, Science 357, 372 (2017).

[13] J. A. Blackmore, L. Caldwell, P. D. Gregory, E. M. Bridge, R. Sawant, J. Aldegunde, J. Mur-Petit, D. Jaksch, J. M. Hutson, B. E. Sauer et al., Quantum Sci. Technol. 4, 014010 (2018).

[14] S. N. Vogels, J. Onvlee, S. Chefdeville, A. van der Avoird, G. C. Groenenboom, and S. Y. T. van de Meerakker, Science 350, 787 (2015).

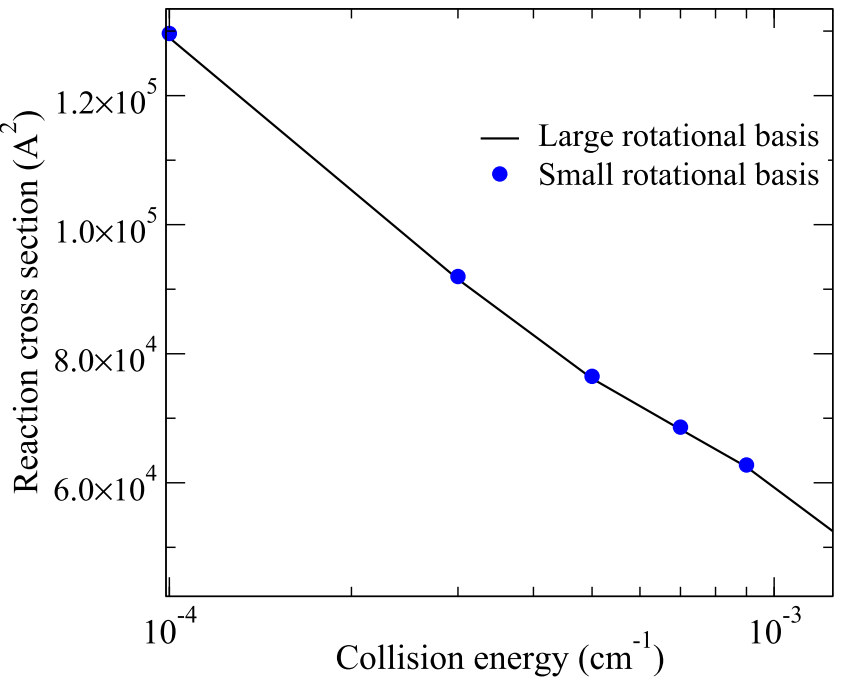

FIG. 6. Total CCS reaction cross sections calculated using large $\left(N_{\max }=55\right.$, black line $)$ and small $\left(N_{\max }=2\right.$, circles $)$ rotational basis sets for the initial spin states $\left|\frac{1}{2},-\frac{1}{2}\right\rangle_{\mathrm{Li}}+\left|\frac{1}{2}, \frac{1}{2}\right\rangle_{\mathrm{CaH}}$ in the absence of the hyperfine structure. The magnetic field is $B=0.01 \mathrm{~T}$ and $J_{\max }=2$.

The large rotational basis sets are required to account for the large anisotropy of the $\mathrm{Li}-\mathrm{CaH}$ interaction, as shown in our previous work on nonreactive spin relaxation in ultracold Li-CaH collisions [56].

[15] S. N. Vogels, T. Karman, J. Kłos, M. Besemer, J. Onvlee, A. van der Avoird, G. C. Groenenboom, and S. Y. T. van de Meerakker, Nat. Chem. 10, 435 (2018).

[16] K.-K. Ni, S. Ospelkaus, D. Wang, G. Quéméner, B. Neyenhuis, M. H. G. de Miranda, J. L. Bohn, J. Ye, and D. S. Jin, Nature (London) 464, 1324 (2010).

[17] X. Ye, M. Guo, M. L. González-Martínez, G. Quéméner, and D. Wang, Sci. Adv. 4, eaaq0083 (2018).

[18] H. Yang, D.-C. Zhang, L. Liu, Y.-X. Liu, J. Nan, B. Zhao, and J.-W. Pan, Science 363, 261 (2019).

[19] S. Ospelkaus, K.-K. Ni, D. Wang, M. H. G. de Miranda, B. Neyenhuis, G. Quéméner, P. S. Julienne, J. L. Bohn, D. S. Jin, and J. Ye, Science 327, 853 (2010).

[20] M. H. G. de Miranda, A. Chotia, B. Neyenhuis, D. Wang, G. Quéméner, S. Ospelkaus, J. L. Bohn, J. Ye, and D. S. Jin, Nat. Phys. 7, 502 (2011).

[21] J. P. J. Driessen, H. J. L. Megens, M. J. Zonneveld, H. A. J. Senhorst, H. C. W. Beijerinck, and B. J. Verhaar, Chem. Phys. 147, 447 (1990).

[22] D. Watanabe, H. Ohoyama, T. Matsumura, and T. Kasai, Phys. Rev. Lett. 99, 043201 (2007).

[23] S. D. S. Gordon, J. J. Omiste, J. Zou, S. Tanteri, P. Brumer, and A. Osterwalder, Nat. Chem. 10, 1190 (2018).

[24] E. S. Shuman, J. F. Barry, and D. DeMille, Nature (London) 467, 820 (2010).

[25] J. F. Barry, D. J. McCarron, E. B. Norrgard, M. H. Steinecker, and D. DeMille, Nature (London) 512, 286 (2014). 
[26] S. Truppe, H. J. Williams, M. Hambach, L. Caldwell, N. J. Fitch, E. A. Hinds, B. E. Sauer, and M. R. Tarbutt, Nat. Phys. 13, 1173 (2017)

[27] L. Anderegg, B. L. Augenbraun, E. Chae, B. Hemmerling, N. R. Hutzler, A. Ravi, A. Collopy, J. Ye, W. Ketterle, and J. M. Doyle, Phys. Rev. Lett. 119, 103201 (2017).

[28] L. Anderegg, B. L. Augenbraun, Y. Bao, S. Burchesky, L. W. Cheuk, W. Ketterle, and J. M. Doyle, Nat. Phys. 14, 890 (2018).

[29] L. W. Cheuk, L. Anderegg, B. L. Augenbraun, Y. Bao, S. Burchesky, W. Ketterle, and J. M. Doyle, Phys. Rev. Lett. 121, 083201 (2018).

[30] D. J. McCarron, M. H. Steinecker, Y. Zhu, and D. DeMille, Phys. Rev. Lett. 121, 013202 (2018).

[31] J. Lim, J. R. Almond, M. A. Trigatzis, J. A. Devlin, N. J. Fitch, B. E. Sauer, M. R. Tarbutt, and E. A. Hinds, Phys. Rev. Lett. 120, 123201 (2018)

[32] I. Kozyryev, L. Baum, K. Matsuda, B. L. Augenbraun, L. Anderegg, A. P. Sedlack, and J. M. Doyle, Phys. Rev. Lett. 118, 173201 (2017).

[33] T. V. Tscherbul and R. V. Krems, Phys. Rev. Lett. 97, 083201 (2006).

[34] E. Abrahamsson, T. V. Tscherbul, and R. V. Krems, J. Chem. Phys. 127, 044302 (2007).

[35] S. Knoop, F. Ferlaino, M. Berninger, M. Mark, H.-C. Nägerl, R. Grimm, J. P. D’Incao, and B. D. Esry, Phys. Rev. Lett. 104, 053201 (2010).

[36] J.-L. Li and S.-L. Cong, Phys. Rev. A 99, 022708 (2019).

[37] L. M. C. Janssen, A. van der Avoird, and G. C. Groenenboom, Phys. Rev. Lett. 110, 063201 (2013).

[38] E. J. Rackham, F. Huarte-Larranaga, and D. E. Manolopoulos, Chem. Phys. Lett. 343, 356 (2003).

[39] E. J. Rackham, T. Gonzalez-Lezana, and D. E. Manolopoulos, J. Chem. Phys. 119, 12895 (2003).

[40] M. H. Alexander, E. J. Rackham, and D. E. Manolopoulos, J. Chem. Phys. 121, 5221 (2004).

[41] T. González-Lezana, Int. Rev. Phys. Chem. 26, 29 (2007).

[42] W. Miller, J. Chem. Phys. 52, 543 (1970).

[43] D. C. Clary, Annu. Rev. Phys. Chem. 41, 61 (1990).

[44] G. Quéméner and J. L. Bohn, Phys. Rev. A 81, 022702 (2010).

[45] M. L. T. González-Martínez, O. Dulieu, P. Larrégaray, and L. Bonnet, Phys. Rev. A 90, 052716 (2014).

[46] Z. Idziaszek and P. S. Julienne, Phys. Rev. Lett. 104, 113202 (2010).

[47] B. Gao, Phys. Rev. Lett. 105, 263203 (2010).

[48] G. Quéméner and P. S. Julienne, Chem. Rev. 112, 4949 (2012).

[49] S. Kotochigova, New J. Phys. 12, 073041 (2010).

[50] P. J. Dagdigian, J. Chem. Phys. 146, 224308 (2017).

[51] J. F. E. Croft, C. Makrides, M. Li, A. Petrov, B. K. Kendrick, N. Balakrishnan, and S. Kotochigova, Nat. Commun. 8, 15897 (2017).

[52] C. Makrides, J. Hazra, G. B. Pradhan, A. Petrov, B. K. Kendrick, T. González-Lezana, N. Balakrishnan, and S. Kotochigova, Phys. Rev. A 91, 012708 (2015).

[53] T. V. Tscherbul and A. A. Buchachenko, New J. Phys. 17, 035010 (2015).
[54] V. Singh, K. S. Hardman, N. Tariq, M.-J. Lu, A. Ellis, M. J. Morrison, and J. D. Weinstein, Phys. Rev. Lett. 108, 203201 (2012).

[55] L. D. Carr, D. DeMille, R. V. Krems, and J. Ye, New J. Phys. 11, 055049 (2009).

[56] T. V. Tscherbul, J. Kłos, and A. A. Buchachenko, Phys. Rev. A 84, 040701(R) (2011).

[57] J. Lim, M. D. Frye, J. M. Hutson, and M. R. Tarbutt, Phys. Rev. A 92, 053419 (2015).

[58] M. Morita, M. B. Kosicki, P. S. Żuchowski, and T. V. Tscherbul, Phys. Rev. A 98, 042702 (2018).

[59] T. V. Tscherbul and A. Dalgarno, J. Chem. Phys. 133, 184104 (2010).

[60] G. C. Groenenboom and L. M. C. Janssen, in Tutorials in Molecular Reaction Dynamics, edited by M. Brouard and C. Vallance (Royal Society of Chemistry, Cambridge, 2010).

[61] A. J. Moerdijk, B. J. Verhaar, and T. M. Nagtegaal, Phys. Rev. A 53, 4343 (1996).

[62] T. Sikorsky, M. Morita, Z. Meir, A. A. Buchachenko, R. Benshlomi, N. Akerman, E. Narevicius, T. V. Tscherbul, and R. Ozeri, Phys. Rev. Lett. 121, 173402 (2018).

[63] M. Warehime and J. Kłos, Phys. Rev. A 92, 032703 (2015).

[64] P. Puri, M. Mills, C. Schneider, I. Simbotin, J. A. Montgomery, R. Côté, A. G. Suits, and E. R. Hudson, Science 357, 1370 (2017).

[65] T. Yang, A. Li, G. K. Chen, C. Xie, A. G. Suits, W. C. Campbell, H. Guo, and E. R. Hudson, J. Phys. Chem. Lett. 9, 3555 (2018).

[66] D. Zhang and S. Willitsch, in Cold Chemistry: Molecular Scattering and Reactivity near Absolute Zero, edited by O. Dulieu and A. Osterwalder (Royal Society of Chemistry, Cambridge, 2017).

[67] F. H. J. Hall and S. Willitsch, Phys. Rev. Lett. 109, 233202 (2012).

[68] E. R. Meyer and J. L. Bohn, Phys. Rev. A 83, 032714 (2011).

[69] J. F. E. Croft, A. O. G. Wallis, J. M. Hutson, and P. S. Julienne, Phys. Rev. A 84, 042703 (2011).

[70] A. Volpi and J. L. Bohn, Phys. Rev. A 65, 052712 (2002).

[71] R. V. Krems and A. Dalgarno, J. Chem. Phys. 120, 2296 (2004).

[72] T. V. Tscherbul, J. Kłos, L. Rajchel, and R. V. Krems, Phys. Rev. A 75, 033416 (2007).

[73] D. E. Manolopoulos (unpublished).

[74] T. V. Tscherbul, in Cold Chemistry: Molecular Scattering and Reactivity near Absolute Zero, edited by O. Dulieu and A. Osterwalder (Royal Society of Chemistry, Cambridge, 2017), Chap. 6.

[75] H.-J. Werner, P. J. Knowles, G. Knizia, F. R. Manby, and M. Schütz, WIREs Comput. Mol. Sci. 2, 242 (2012).

[76] P. J. Knowles, C. Hampel, and H.-J. Werner, J. Chem. Phys. 99, 5219 (1993).

[77] H. Werner and P. J. Knowles, J. Chem. Phys. 89, 5803 (1988).

[78] T. H. Dunning, J. Chem. Phys. 90, 1007 (1989).

[79] J. Koput and K. A. Peterson, J. Phys. Chem. A 106, 9595 (2002).

[80] T.-S. Ho and H. Rabitz, J. Chem. Phys. 104, 2584 (1996). 